\title{
Effect of metallic nanoparticle fillers on the thermal conductivity of diatomaceous earth
}

Mouhamad S. Diallo ${ }^{1,3}$, Srilok Srinivasan ${ }^{2}$, Boyce Chang ${ }^{3}$, Suvojit Ghosh ${ }^{4}$, and Ganesh Balasubramanian, ${ }^{2, *}$

${ }^{1}$ Department of Liberal Arts, Des Moines Area Community College, Des Moines, IA 50314, USA

${ }^{2}$ Department of Mechanical Engineering, Iowa State University, Ames, IA 50011, USA

${ }^{3}$ Department of Materials Science and Engineering, Iowa State University, Ames, IA 50011, USA

${ }^{4}$ Department of Engineering Physics, McMaster University, Hamilton, ON L8S4L8, Canada

* Corresponding author Department of Mechanical Engineering, 2092 Black Engineering, Iowa State University, Ames, IA 50011, USA Tel.: +1 -515-294-9226

Email: bganesh@iastate.edu 


\begin{abstract}
Thermal conductivity of solid nanoparticles (aluminum) in a nanoporous solid matrix (diatomaceous earth) is examined to understand the effect of conductive fillers on the thermal properties of a porous material. We find that thermal conductivity is strongly dependent on load applied to prepare the mixture compacts, while porosity is influenced by the composition of the mixture. The addition of nanoparticles contributes to limited increases in thermal conductivity of the mixture by (1) increasing contact area between the mixture constituents and (2) reduction of porosity that leads to enhanced solid-gas coupling contribution. Thermal conductivity increases exponentially with external gas pressures due to the coupling effect between the solid particles and the entrapped air.
\end{abstract}

\title{
Keywords
}

Aluminum nanoparticles, Diatomaceous earth, Thermal conductivity, Porosity 


\section{Introduction}

Diatomaceous earth (DE) is a naturally occurring soft powdered material composed of fossilized remains of unicellular algae called diatoms; up to 100,000 species of diatoms can be found in a single sample ${ }^{1-3}$. The diatom skeletons are primarily made of amorphous silica. Each of the different diatom species has a unique frustule structure, making DE an extremely heterogeneous material with notable variations in physical properties ${ }^{2,4-6}$. DE finds applications as filters, building material, abrasive, catalyst carrier, acoustic and thermal insulators, and even as ingredients in some medicines ${ }^{7-12}$. DE has a low thermal conductivity $(\sim 0.1 \mathrm{~W} / \mathrm{mK})$ because of its nanoporous structure, and has recently received interest as a potential candidate for core insulation material in superior performance vacuum insulation panels (VIPs) ${ }^{8,13-14}$. The vacuum inside VIPs degrades with time due to pressure difference, ultimately resulting in gradual loss of thermal resistance. A major challenge is to increase the lifetime of VIPs for use in energy efficient buildings, and one possible solution is to tune the thermal conductivity of DE with fillers. While thermal conductivity $(k)$ of pure DE has been reported in the literature 7,15 , the effect of impregnating the nanoscale pores with nanoparticles has not been explored. In particular, the presence of thermally conductive nanoscale fillers in the DE porous matrix can facilitate application-specific modification of $k$ for insulation materials. Furthermore, DE has been traditionally used as a high temperature insulator $\left(>1000^{\circ} \mathrm{C}\right)$ whereby radiation heat transfer becomes especially pronounced in porous material 3, 14. Since metallic nanofillers could function as an opacifier without significantly increasing the thermal conductivity of the mixture, we examine the influence of Al nanoparticles (AINPs) dispersed in bulk DE on the $k$ of the mixture.

The thermal conductivity of porous materials depends, as obvious, on porosity ${ }^{16-20}$. In addition, $k$ of granular materials like DE is strongly influenced by the contact resistance between particles, leading to a significant change in $k$ with gas pressure ${ }^{8}$. In this letter, we discuss the contribution of nanoparticles and porosity on the $k$ of AlNP/DE mixture. The addition of AlNPs, as expected, increases the overall $k$ of the mixture. However, as discussed below in details, this increment is only marginal even though a highly conductive material such as $\mathrm{Al}$ is used. Our results aid in understanding how the fillers influence $k$ by modifying the contact between the mixture components. 


\section{Materials and Methods}

AlNPs of $\sim 800 \mathrm{~nm}$ diameter (US Research Nanomaterials, Inc) are mixed with DE having a pore size of $\sim 1 \mu \mathrm{m}$ (Perma-Guard ${ }^{\mathrm{TM}}$, Inc) by shaking in a vibratory mill for $6 \mathrm{~h}$, followed by dry pressing to form cylindrical compacts. The density of each compact is determined by measuring the mass and volume. In total, twenty-four samples are prepared each with varying AlNP mass fraction ( $0 \%, 10 \%, 20 \%, 30 \%, 40 \%$, and $50 \%)$ and compaction load (0.45 MPa, 1.13 MPa, 1.70MPa, 2.26 MPa). $k$ is measured by the transient plane source technique (Hot Disk ${ }^{\mathrm{TM}}$ TPS 1500) that employs a sensor acting as both a heat source and a resistance thermometer. The change in temperature is measured as a function of time, and subsequently $k$ is obtained using the Gustafsson equation ${ }^{21}$. The microstructure of the samples is examined under a scanning electron microscope (SEM, JEOL JSM-6060LV). For two of the samples, viz., pure DE and 50\% (w/w) AlNP/DE mixture, we measure $k$ as a function of gas pressure between $27 \mathrm{~Pa}(\sim$ vacuum) to $10^{5} \mathrm{~Pa}(\sim 1 \mathrm{~atm}$.), by evacuating the sample in a bell jar (schematic shown in Figure 1).

\section{Results and Discussion}

Effect of nanoparticle concentration: Air occupying the nanopores in pure DE at atmospheric pressure contributes to the overall heat conduction ${ }^{22}$. With the addition of AlNPs, these air pockets are replaced by highly conductive metallic particles. We neglect the influence of nanoscale size effects since both the electron mean free path $\left(\lambda_{\text {electron }} \sim 22\right.$ $\mathrm{nm})^{23}$ and the phonon mean free path $\left(\lambda_{\text {phonon }} \sim 8.1 \mathrm{~nm}\right){ }^{24}$ of $\mathrm{Al}$ are much smaller than the average particle diameter of $800 \mathrm{~nm}$. Thus, $k_{A l}$ for the nanoparticle used in this investigation is similar to the bulk Al thermal conductivity $\left(k_{\text {bulk }}=237 \mathrm{~W} / \mathrm{m} . \mathrm{K}\right)$.

We present the change in effective $k$ of the mixture with AlNP concentration in Fig. 2a. Though $k_{A l}$ is significantly higher than that of pure DE, $k$ for the mixture increases only by $15 \%$ even for $50 \%$ nanoparticle mass fraction under a compaction of $2.26 \mathrm{MPa}$. We conjecture that this small increase in $k$ is due to impedance to heat transfer by interfacial thermal resistance arising from the increase in interfacial areas between dissimilar surfaces of $\mathrm{Al}$ and $\mathrm{DE}$ particles. Considering an interfacial thermal conductance $\mathrm{G}=120$ $\mathrm{MW} / \mathrm{m}^{2} \mathrm{~K}{ }^{25}$ between the Al nanoparticles and DE, the Kapitza length $\left(L_{K}\right)$ calculated with the bulk thermal conductivity of $\mathrm{Al}$, is $\sim 1.975 \mu \mathrm{m}$. This $L_{K}$ is much larger than the 
average Al nanoparticle size $(800 \mathrm{~nm})$ employed here, suggesting that interfacial thermal resistance exerts a significant effect on heat conduction in the AINP/DE mixture. The results also suggest that compaction has a more pronounced effect on $k$ as compared against the influence of mixture composition. Increased load during dry pressing results in mixtures that are more compact with less voids between material particles, eliminating the air trapped within the interfacial areas, and thus increasing the effective $k$.

A higher compaction pressure creates structures with lower porosity. Porosity is related to both the gaseous molecules in the interfacial zones as well as the "coupling effect". The coupling effect takes into account heat transfer between solid and gas phases when modeling the effective thermal conductivity of porous materials such as aerogels ${ }^{26-29}$. Effective thermal conductivity is typically determined by considering a combination of solid, gaseous and the coupling contributions to thermal conductivity. The coupling contribution is approximated as the composite of both solid and gas phases (proportional to their volume fractions) connected in series along the direction of heat flow, resulting in an intermediate $k$. Thus, lower porosity results in greater solid phase contribution to the coupling effect, which leads to higher measured thermal conductivity.

Accordingly, as shown in Figs. $2 \mathrm{a}$ and $2 \mathrm{~b}$, a lower porosity contributes to a relatively higher $k$. Porosities $(\Phi)$ are predicted from the densities of the mixtures as $\Phi=1-$ $\frac{\rho_{\text {avg }}}{\rho_{\text {effective }}}$, where $\rho_{\text {avg }}$ is the measured mean density and $\rho_{\text {effective }}=\rho_{S}(1-x)+$ $\rho_{A l}(x) . \rho_{S}$ is density of silica, $\rho_{A l}$ is density of bulk Al and $x$ is concentration of AlNPs. $\Phi$ of the mixture is dependent on both the nanoparticle mass fraction and the compaction load used. An empirical fit of data presented in Fig. $2 \mathrm{~b}$ gives $\Phi=0.7594-0.1852 x-$ $0.02787 C_{p}$, where $C_{p}$ is the compaction pressure. This dependence of $\Phi$ on $x$ and $C_{p}$ shows that, contrary to $k$, porosity is more sensitive to composition than compaction, and hence is only weakly correlated to the effective $k$ of the mixture. Although the addition of AlNP leads to a larger change in the effective density of composite, the change in $k$ is very small (Fig. 2c). On the other hand, despite the small increase in density with increasing compaction, we can observe a relatively large change in $k$ for each of the AlNP concentrations considered. 
Fig. 3a shows that $k$ for both pure DE (with 0\% AlNP) and DE containing 50\% nanoparticles increases with increasing compaction but saturates above 1.70 MPa. The relative difference in $k$ of the two samples widens with stronger compaction. The corresponding results measured under vacuum $(27 \mathrm{~Pa})$ presented in Fig. 3b, reveal a similar trend. Under vacuum conditions, conduction at the contact between solid particles is the predominant mechanism of thermal transport. The similarity between the two results suggests that the addition of AlNPs in DE increases the overall contact area, which increases $k$. Fig. 3c illustrates that the difference in $k$ between the samples $(\Delta k=$ $\left.k_{50 \% A l}-k_{\text {pure } D E}\right)$ at 1 atm and at vacuum are comparable for lower compaction pressures but significantly differ as the applied load is increased. $\Delta k$ at vacuum remains similar for the different compactions, implying that the contribution of AlNPs in increasing the interparticle contact areas is consistent over the range of loads considered. The rise in $\Delta k$ at 1 atm. with increasing compaction is due to the lower porosity and the coupling effect ${ }^{29}$ in the presence of the pockets of air. Analyzing the microstructure of samples with different composition and compaction under SEM, as illustrated in Fig. 4, also confirms that nanoparticles do not undergo any noteworthy deformation.

Our results indicate that the addition of AlNPs does not significantly increase thermal conductivity of the material (Fig. 2a). These particles fill the pores of DE (Fig. 2b and Fig. 4), which is followed by an enhanced coupling effect (Fig. 3). High temperature insulation materials are typically more exposed to radiation heat transfer due to high porosity. Hence, the application of AINP could lead to an improvement in the opacity of the material (by filling pores) without significantly increasing the overall thermal conductivity.

Effect of vacuum: Measurements in vacuum eliminate the contributions of gas conduction and effectively isolate the role of the porous medium. Vacuum generation is also employed to reduce $k$ in high efficiency insulators like VIPs ${ }^{30-35}$. Thermal conductivity measurements with varying gas pressures for pure DE and 50\% AlNP/DE, both prepared at $2.26 \mathrm{MPa}$, are presented in Fig. 5. The difference between the two sets of measurements is attributed to the enhanced solid contact area due to the addition of AlNPs. The increase in $k$ with rise in pressure for the two samples is qualitatively similar. This result is characteristic of conduction by coupling effect since it is strongly dependent 
on the concentration of gas phase that varies with pressure. In vacuum, the mass of gas present in the mixture is minimal and hence its contribution to $k$ is negligible. Heat transfer is predominantly due to conduction through the solid particles and across the contact areas between them. However, as the amount of air inside the porous material increases not only does the contribution due to gas conduction is enhanced but it also gives rise to coupling effect ${ }^{29}$. The mean free path of the gas molecules, $\lambda_{\text {air }}$, is calculated

to be in the range of $64-68 \mathrm{~nm}$ depending on the relative humidity ${ }^{36}$. Although mercury porosimetry measurements reveal that DE contains pores of sizes comparable to the mean free path of gas molecules $(<100 \mathrm{~nm})^{14}$, these constitute less than $5 \%$ of the total porosity. The majority of the pores in DE are of microscopic dimensions. Hence the Knudsen effect associated with gas conduction is negligible in this case. It has been shown for glass fiber fleece with average pore size of $1 \mu \mathrm{m}$ that the coupling effect initiates around $10^{2}-10^{3} \mathrm{~Pa}^{29}$. Here, as shown in Fig. 5 with a log-log distribution, we observe an exponential dependence of $k$ on the external gas pressure.

\section{Summary}

We investigate the thermal conductivity $k$ of mixtures of nanoporous $\mathrm{DE}$ and $\mathrm{Al}$ nanoparticles for different compaction loads, compositions and gas pressures using the transient plane source technique. $k$ is strongly correlated to compaction while only slightly to nanoparticle mass fraction. In contrast, the filler concentration has a pronounced effect on porosity. Measurements in vacuum reveal that addition of AlNPs affects $k$ by increasing the solid contact area rather than through its effect on porosity. Our SEM characterization illustrates no deformations in $\mathrm{Al}$ nanoparticles suggesting that solid contact area does not change with compaction. However, as the air content in the mixture increases, $k$ increases exponentially with rising gas pressures aided by the solidgas coupling effect. Our results contribute to improved understanding of the thermal properties of nanoscale solid dispersions in a porous matrix where the constituent species have significant differences in thermal conductivities. The mixture investigated in this work has potential applications for high temperature insulation materials where radiation heat transfer can become important. 


\section{Acknowledgements}

The research is supported, in part, through the National Science Foundation (NSF) grant no. CMMI-1404938. The authors thank the Iowa State University MOSAIC REU program supported through the NSF award EEC-1263243, the Iowa Energy Center grant no. 13-04, the Des Moines Area Community College LSAMP-IINSPIRE (supported by NSF grant no. HD-1102461, 2011-2016) for partially funding this project. We thank Prof. Mufit Akinc for the use of his lab facilities. 


\section{References}

1. Losic, D.; Mitchell, J. G.; Voelcker, N. H., Diatomaceous Lessons in Nanotechnology and Advanced Materials. Advanced Materials 2009, 21, 2947-2958.

2. Round, F. E.; Crawford, R. M.; Mann, D. G., The Diatoms: Biology \& Morphology of the Genera; Cambridge University Press, 1990.

3. Calvert, R., Diatomaceous Earth. Journal of Chemical Education 1930, 7, 2829.

4. $\quad$ Butcher, K. S. A.; Ferris, J. M.; Phillips, M. R.; Wintrebert-Fouquet, M.; Jong Wah, J. W.; Jovanovic, N.; Vyverman, W.; Chepurnov, V. A., A Luminescence Study of Porous Diatoms. Materials Science and Engineering: C 2005, 25, 658-663.

5. Hamm, C. E.; Merkel, R.; Springer, O.; Jurkojc, P.; Maier, C.; Prechtel, K.; Smetacek, V., Architecture and Material Properties of Diatom Shells Provide Effective Mechanical Protection. Nature 2003, 421, 841-843.

6. Losic, D.; Short, K.; Mitchell, J. G.; Lal, R.; Voelcker, N. H., AFM Nanoindentations of Diatom Biosilica Surfaces. Langmuir 2007, 23, 5014-5021.

7. Ivanov, S. É.; Belyakov, A. V., Diatomite and Its Applications. Glass Ceram 2008, 65, 48-51.

8. Fricke, J.; Heinemann, U.; Ebert, H. P., Vacuum Insulation Panels-from Research to Market. Vacuum 2008, 82, 680-690.

9. Yurkov, A. L.; Aksel'rod, L. M., Properties of Heat-Insulating Materials (a Review). Refract Ind Ceram 2005, 46, 170-174.

10. Ünal, O.; Uygunoğlu, T.; Yildiz, A., Investigation of Properties of Low-Strength Lightweight Concrete for Thermal Insulation. Building and Environment 2007, 42, 584590.

11. Karaman, S.; Karaipekli, A.; Sarı, A.; Biçer, A., Polyethylene Glycol (Peg)/Diatomite Composite as a Novel Form-Stable Phase Change Material for Thermal Energy Storage. Solar Energy Materials and Solar Cells 2011, 95, 1647-1653.

12. Davis, L., Diatomite; American Ceramic Society: Westerville, OH, 1991;70, 2.

13. Fricke, J.; Schwab, H.; Heinemann, U., Vacuum Insulation Panels - Exciting Thermal Properties and Most Challenging Applications. Int J Thermophys 2006, 27, 1123-1139.

14. Chang, B.; Zhong, L.; Akinc, M., Low Cost Composites for Vacuum Insulation Core Material. Vacuum 2016, 131, 120-126.

15. Niven, C. D., On the Thermal Conductivity of Diatomite at Moderately High Temperatures. Canadian Journal of Research 1934, 11, 249-253.

16. Smith, D. S.; Alzina, A.; Bourret, J.; Nait-Ali, B.; Pennec, F.; Tessier-Doyen, N.; Otsu, K.; Matsubara, H.; Elser, P.; Gonzenbach, U. T., Thermal Conductivity of Porous Materials. Journal of Materials Research 2013, 28, 2260-2272.

17. Woodside, W.; Messmer, J. H., Thermal Conductivity of Porous Media. I. Unconsolidated Sands. Journal of Applied Physics 1961, 32, 1688-1699.

18. Brailsford, A. D.; Major, K. G., The Thermal Conductivity of Aggregates of Several Phases, Including Porous Materials. British Journal of Applied Physics 1964, 15, 313.

19. Carson, J. K.; Lovatt, S. J.; Tanner, D. J.; Cleland, A. C., Thermal Conductivity Bounds for Isotropic, Porous Materials. International Journal of Heat and Mass Transfer 2005, 48, 2150-2158. 
20. Machrafi, H.; Lebon, G., Size and Porosity Effects on Thermal Conductivity of Nanoporous Material with an Extension to Nanoporous Particles Embedded in a Host Matrix. Physics Letters A 2015, 379, 968-973.

21. Gustafsson, S. E., Transient Plane Source Techniques for Thermal Conductivity and Thermal Diffusivity Measurements of Solid Materials. Review of Scientific Instruments 1991, 62, 797-804.

22. Luikov, A. V.; Shashkov, A. G.; Vasiliev, L. L.; Fraiman, Y. E., Thermal Conductivity of Porous Systems. International Journal of Heat and Mass Transfer 1968, 11, 117-140.

23. Hanaoka, Y.; Hinode, K.; Takeda, K. i.; Kodama, D., Increase in Electrical Resistivity of Copper and Aluminum Fine Lines. Materials Transactions 2002, 43, 16211623.

24. Zhou, Y.; Anglin, B.; Strachan, A., Phonon Thermal Conductivity in Nanolaminated Composite Metals Via Molecular Dynamics. Journal of Chemical Physics 2007, 127, 184702.

25. Liu, J.; Zhu, J.; Tian, M.; Gu, X.; Schmidt, A.; Yang, R., Simultaneous Measurement of Thermal Conductivity and Heat Capacity of Bulk and Thin Film Materials Using Frequency-Dependent Transient Thermoreflectance Method. Review of Scientific Instruments 2013, 84, 034902.

26. Bi, C.; Tang, G. H.; Hu, Z. J.; Yang, H. L.; Li, J. N., Coupling Model for Heat Transfer between Solid and Gas Phases in Aerogel and Experimental Investigation. International Journal of Heat and Mass Transfer 2014, 79, 126-136.

27. Zhao, J.-J.; Duan, Y.-Y.; Wang, X.-D.; Wang, B.-X., Effects of Solid-Gas Coupling and Pore and Particle Microstructures on the Effective Gaseous Thermal Conductivity in Aerogels. Journal of Nanoparticle Research 2012, 14, 1-15.

28. Zeng, S. Q.; Hunt, A.; Greif, R., Transport Properties of Gas in Silica Aerogel. Journal of Non-Crystalline Solids 1995, 186, 264-270.

29. Swimm, K.; Reichenauer, G.; Vidi, S.; Ebert, H. P., Gas Pressure Dependence of the Heat Transport in Porous Solids with Pores Smaller Than $10 \mathrm{Mm}$. Int J Thermophys 2009, 30, 1329-1342.

30. Lyman, B. E.; Neeser, T. A. Vacuum Insulation Panel. 1997.

31. Chang, B. S. K. Vacuum Insulation Panel Core Materials and Modelling the Thermal Conductivity of Granular Materials. Iowa State University Ames, Iowa, 2015.

32. Alotaibi, S. S.; Riffat, S., Vacuum Insulated Panels for Sustainable Buildings: A Review of Research and Applications. International Journal of Energy Research 2014, $38,1-19$.

33. Kim, J.; Song, T., Vacuum Insulation Properties of Glass Wool and Opacified Fumed Silica under Variable Pressing Load and Vacuum Level. International Journal of Heat and Mass Transfer 2013, 64, 783-791.

34. Simmler, H.; Brunner, S., Vacuum Insulation Panels for Building Application: Basic Properties, Aging Mechanisms and Service Life. Energy and Buildings 2005, 37, 1122-1131.

35. Wakili, K.; Bundi, R.; Binder, Effective thermal conductivity of vacuum insulation Panels. Building Research and Information 2004, 32, 293-299. 
36. Jennings, S. G., The Mean Free Path in Air. Journal of Aerosol Science 1988, 19, 159-166. 


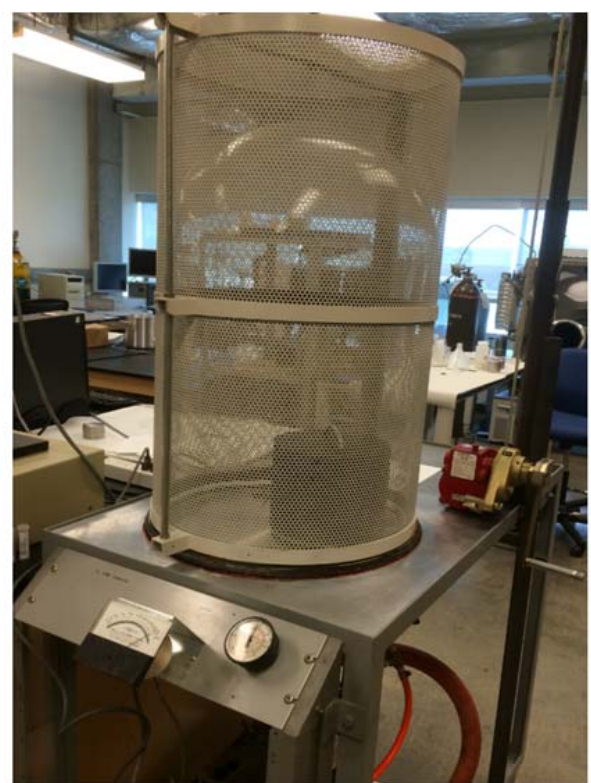

(a)

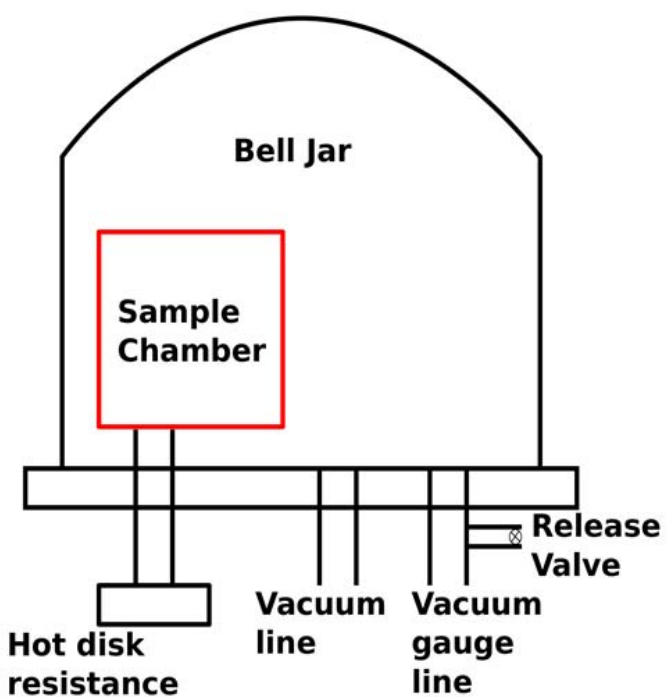

ristance thermometer (sensor)

(b)

\section{Sample}

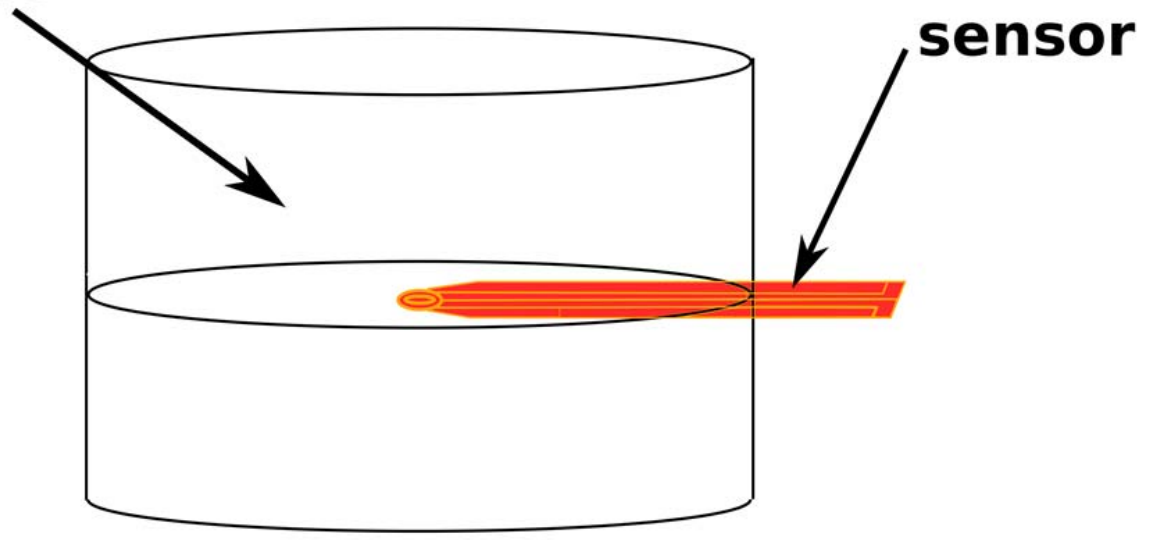

(c)

Figure 1: The experimental setup (a) comprises of a sample chamber placed inside an evacuated bell jar, as shown in the schematic (b). The chamber contains a Hot Disk ${ }^{\mathrm{TM}}$ thermal conductivity probe, which is sandwiched between the dry pressed cylindrical DE samples, as illustrated in (c), during thermal conductivity measurement. 


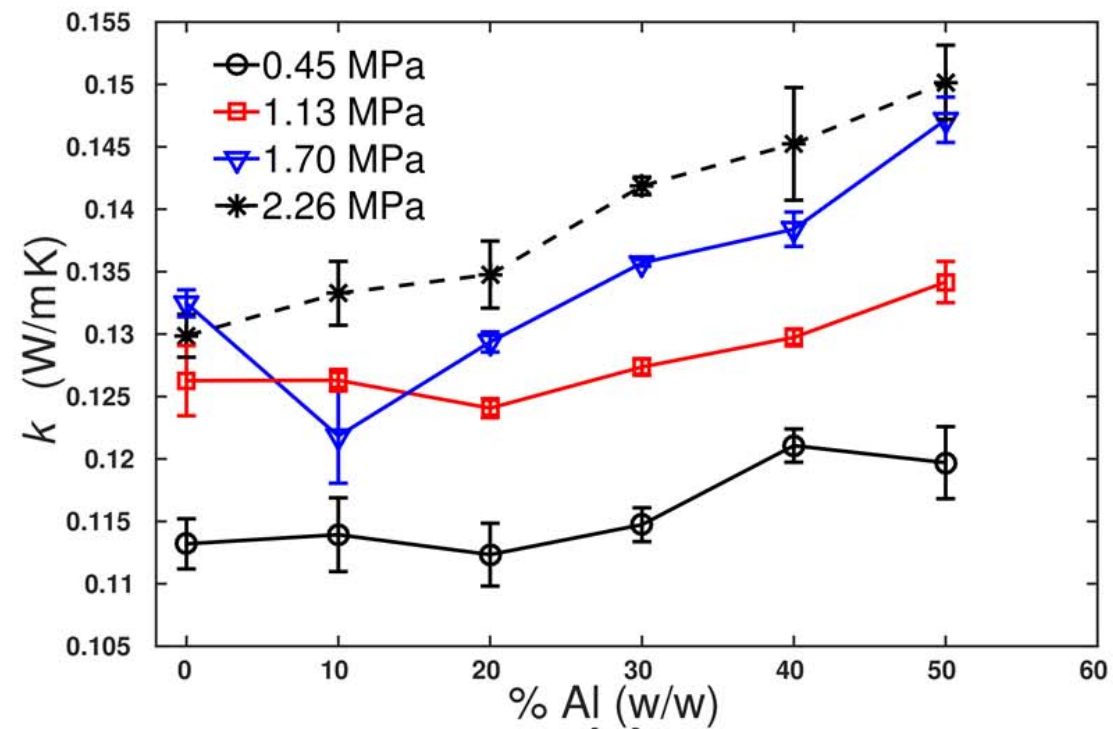

(a)

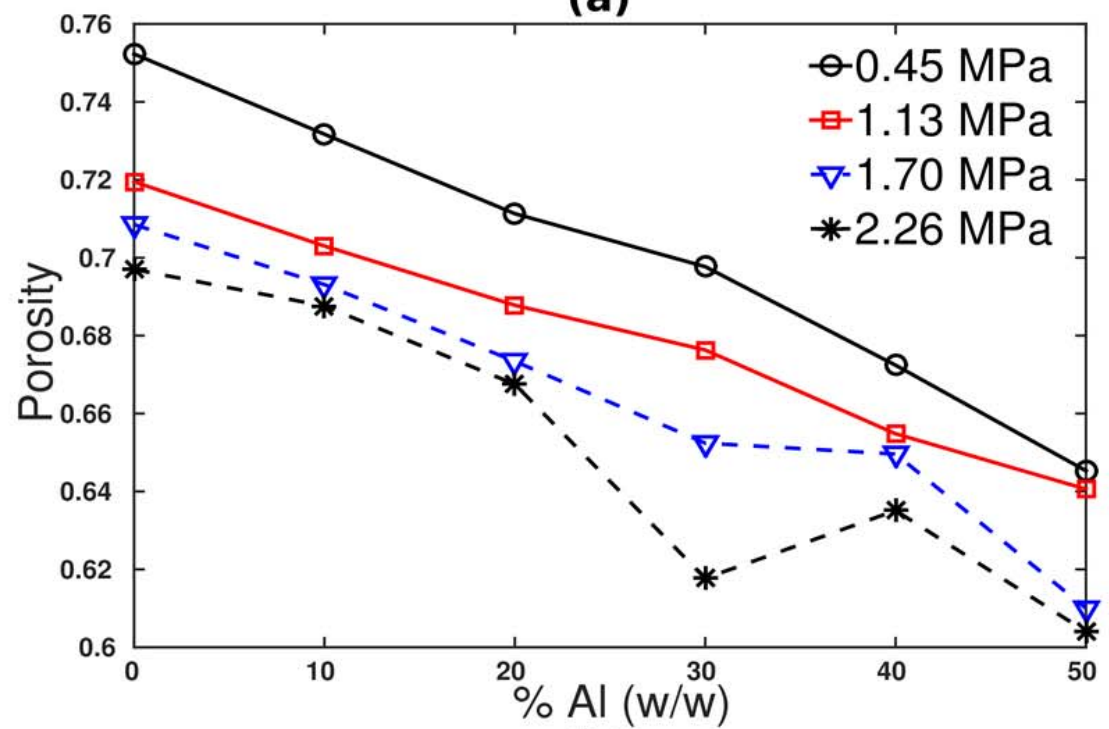

(b)

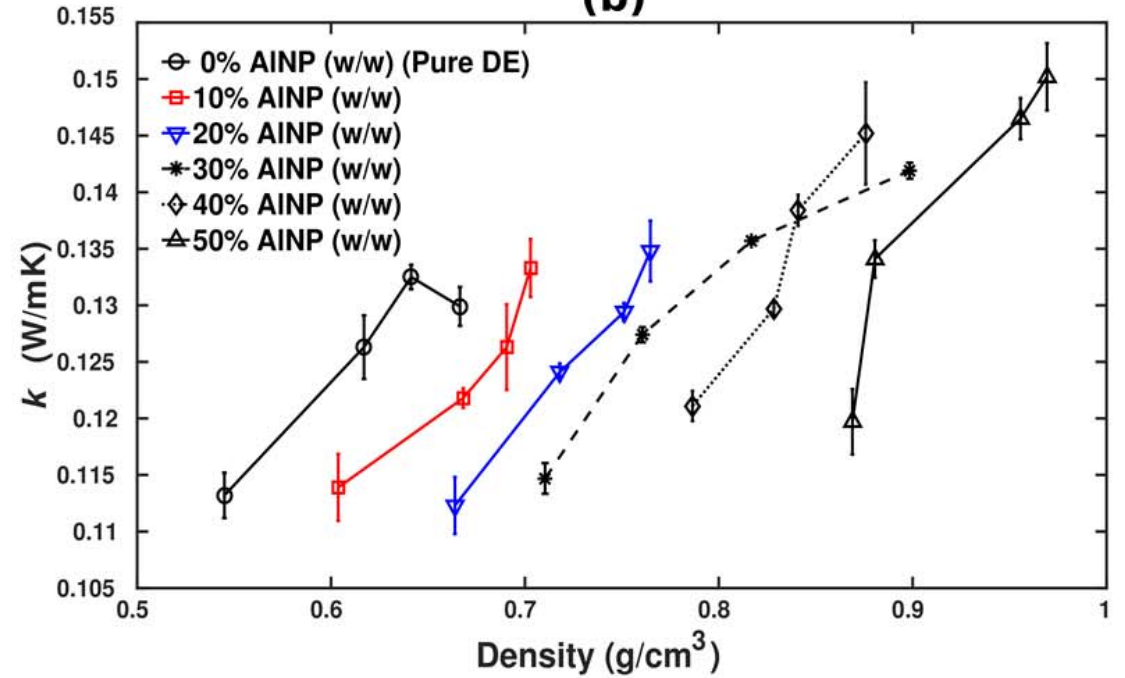

(c) 
Figure 2: (a) The thermal conductivity of AlNP/DE mixtures increase with increases in nanoparticle concentrations and compaction pressures. (b) The strong reduction in porosity with increasing AlNP concentration implies a weak dependence of the overall mixture thermal conductivity on porosity (c) Thermal conductivity variation with density is shown for all the samples. The cases with the same AlNP concentrations are connected by lines to aid data visualization. Despite the large increase in density with addition of AlNP, thermal conductivity is more sensitive to the compaction. 

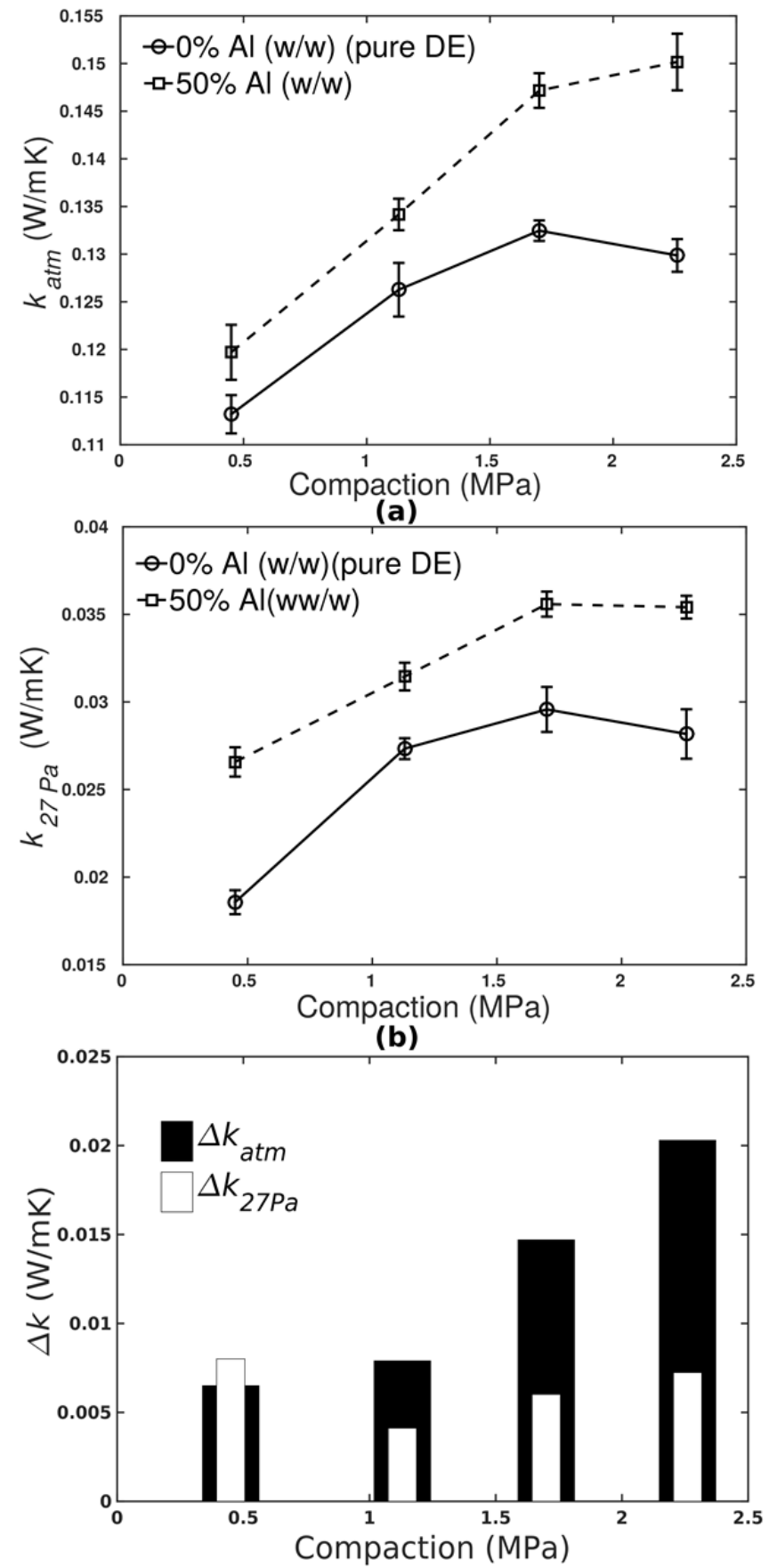

(c)

Figure 3: Thermal conductivity of pure DE and 50\% AlNP/DE mixture is presented for different compaction loads at (a) $10^{5} \mathrm{~Pa}(\sim 1 \mathrm{~atm}$.) and (b) $27 \mathrm{~Pa}(\sim$ vacuum). (c) The difference between thermal conductivity of $50 \%$ AlNP/DE mixture and pure $\mathrm{DE}$ increases with compaction loads, especially at $1 \mathrm{~atm}$., because of the decrease in porosity. 


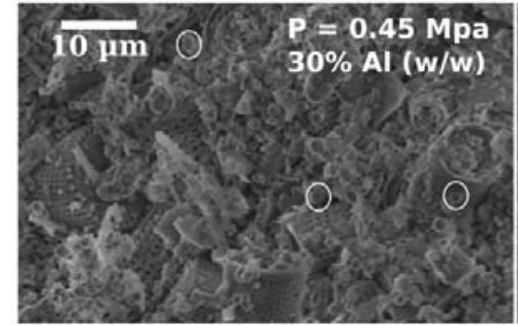

(a)

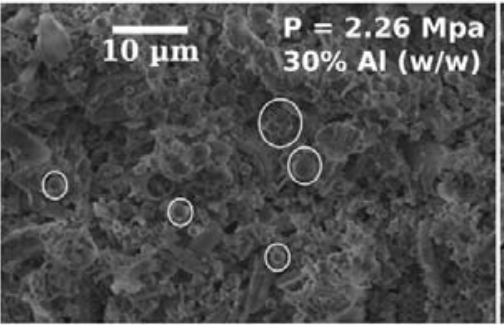

(b)

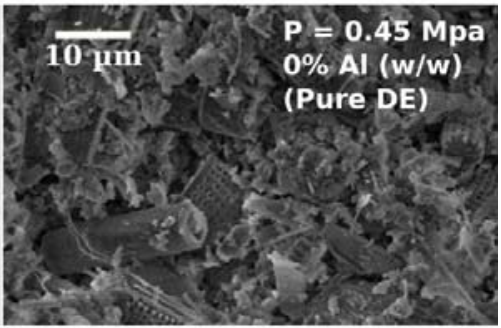

(c)

Figure 4: SEM characterization of the microstructure of the AlNP/DE mixtures at different compactions and nanoparticle concentrations: (a) 30\% AlNP (w/w) at $0.45 \mathrm{MPa}$, (b) $30 \%$ AlNP (w/w) at $2.26 \mathrm{MPa}$, and (c) pure DE at $0.45 \mathrm{MPa}$. No non-negligible deformation in the nanoparticles is observed. 


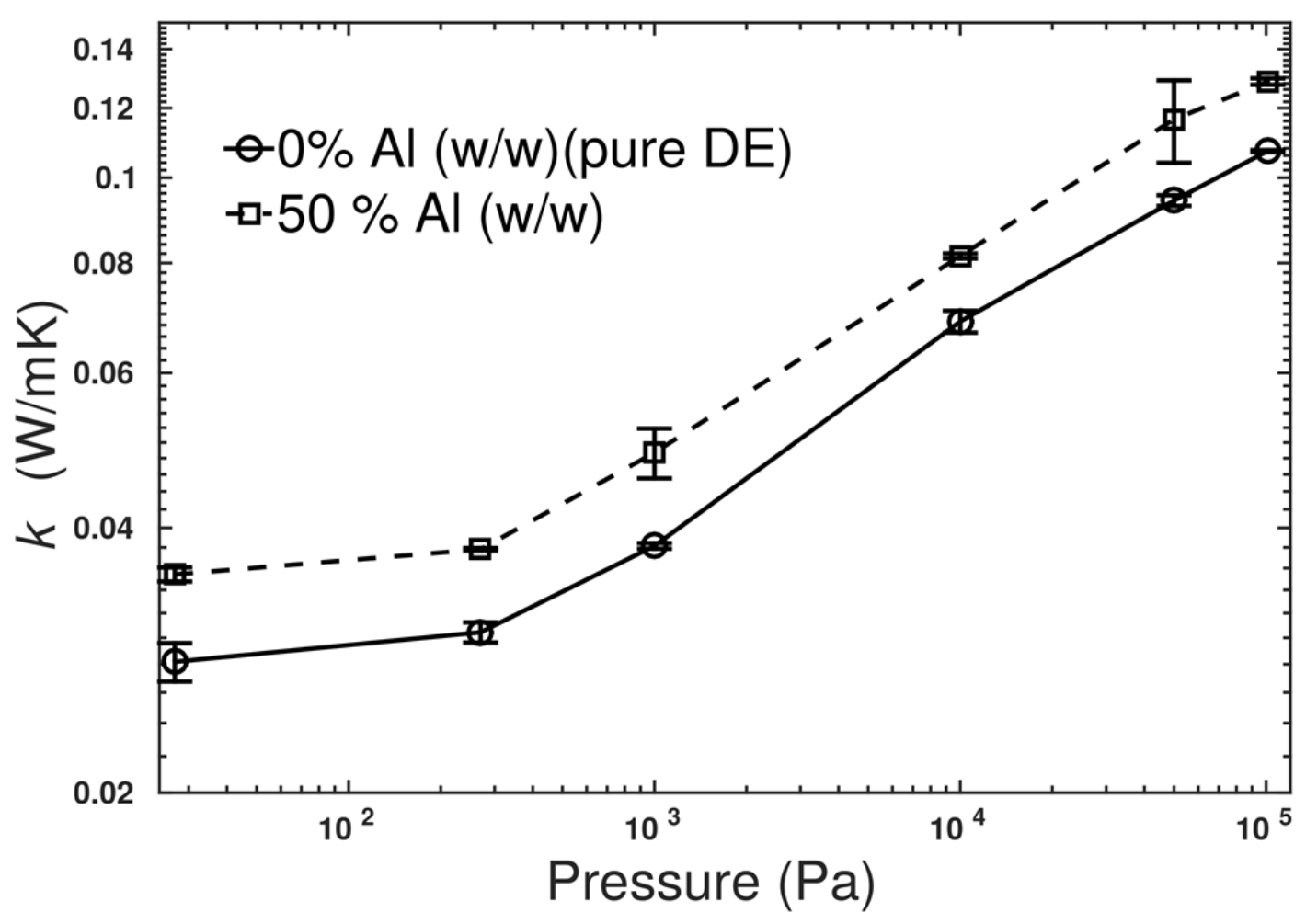

Figure 5: Thermal conductivity of both the pure DE and 50\% AlNP/DE mixture increases with increasing external gas pressure because the air content in the sample pores increases resulting in enhanced contribution of gas conduction to the overall effective thermal conductivity. Additionally, the gas molecules trapped between the DE frustules and nanoparticles induce a coupling effect ${ }^{29}$ due to presence of different material phases resulting in an exponential dependence of thermal conductivity on gas pressure. The difference between the two sets of results is attributed to the enhanced solid contact area due to the addition of AlNP. The data are shown on a set of logarithmic scales. 